\title{
The Simulated Analysis on Shooting Stability of Vehicle Artillery Considering Soil Properties \\ Jie $L I^{1,2, a,{ }^{*}}$ \\ ${ }^{1}$ Mechanical Engineering College, Shjiazhuang, China \\ ${ }^{2}$ No.77, Heiping West Road, Xinhua District, Shijiazhuang City, Hebei Province \\ 15690323252@163.com \\ *Corresponding author
}

Keywords: Vehicle Gun, Soil, Shooting Stability.

\begin{abstract}
Shooting stability is an important factor in the performance of wheeled vehicle artillery combat, has always been the focus of the artillery designers. The mechanical properties of the soil are the important factors that affect the stability of the artillery. In this paper, the vibration problem of the artillery under different soil conditions is studied based on the dynamic model of a car cannon.
\end{abstract}

\section{Introduction}

Car artillery cleverly combines the traditional traction artillery and general wheeled truck chassis, and equipped with electrical, hydraulic and other automation equipment, effectively improve the artillery automation, information, digital level. As the traction artillery structure is simple, recoil through the big frame and other anti-back device to pass to the ground through a single force transmission path. Therefore, the traditional traction artillery has a good shooting stability than the vehicle artillery. In addition, compared with the crawler-type self-propelled artillery, wheeled vehicle guns own lighter weight, larger shooting artillery disturbance.

The mechanical properties of soil materials are complex and changeable. The soil pressure characteristics of the firing environment have a great influence on the recoil distance and the muzzle vibration. Therefore, based on the multi-body system dynamics software ADAMS, a virtual prototyping model of a vehicle-mounted howitzer is established. By changing the mechanical parameters of the contact soil, the vibration of the artillery under different soil conditions is analyzed, and the influence of soil on the shooting stability of vehicle artillery was studied.

\section{Analysis of Soil Properties}

During the firing process, the gunpowder gas burns to produce a strong chamber pressure. In the the action of gun bore force and recoil resistance, the gun body implements recoil movement along the cradle, strong recoil kinetic energy is applied to the gun frame after consumption and absorption the anti-recoil device ,and final to the soil by the hydraulic hoe and seat Plate. As a result, a certain elastic-plastic deformation of the soil is generated ${ }^{[1]}$.

Soil as an uneven, non-continuous material, including solid, gas, liquid three components, the deformation of the situation is more complex ${ }^{[2]}$. According to the classical pressure model(Bekker model), the pressure - subsidence curve of soil under repeated loading and unloading conditions is shown in Fig.1. 


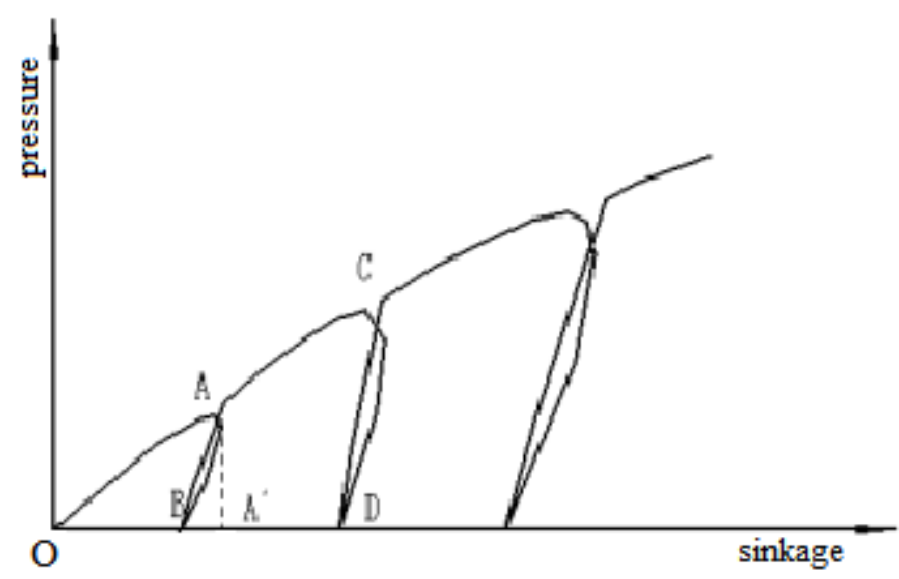

Fig.1 Pressure characteristics of soil

The curve OA segment describes the deformation of the soil at the initial loading. According to the Bekker model, the relationship between soil load and soil subsidence can be expressed as:

$$
p=\left(\frac{k_{c}}{b}+k_{\varphi}\right) z^{n}=k z^{n}
$$

Where: $k_{c}$-- the soil viscosity of the viscous deformation modulus;

$k_{\varphi}$-- the soil friction deformation modulus;

$b$-- the pressure plate width or diameter;

$z$-- soil subsidence.

The $\mathrm{AB}$ segment of the curve describes the unloading deformation of the soil. When the deformation calculation is carried out, the $\mathrm{AB}$ segment is treated as a straight line according to the Wong model. The expression is expressed as:

$$
p=p_{u}-k_{u}\left(z-z_{u}\right)
$$

Where: $k_{u}$--the average slope of the curve $\mathrm{AB}$ segment;

$p_{u}{ }^{--}$the load of the soil before unloading;

$z_{u}$--the subsidence of the soil before unloading.

In this paper, the influence of soil on the vibration of the body is only considered the conditions of once shooting. The soil is only once stressed. Therefore, this paper uses a spring damper to simulate soil deformation, by changing the spring stiffness and damping size to approximate the different nature of the soil.

\section{Dynamics Modeling}

Car cannon is composed of the fire system, fire control system, chassis system and the overall unit .The truthful description of the gun is more difficult because of its complex structure. Therefore, this paper establishes the simplified prototype model of the vehicle-mounted gun according to the main structural features.

\section{Topological Analysis}

In the picture, the chassis part includes the body, the wheel, the ammunition box distribution box, etc. The canister body includes the barrel, the muzzle brake, the breech bar, the retreat pole and the re-entry pole and so on. Thus the entire vehicle gun system is simplified to a 12bodys and 6 degree of freedom . 


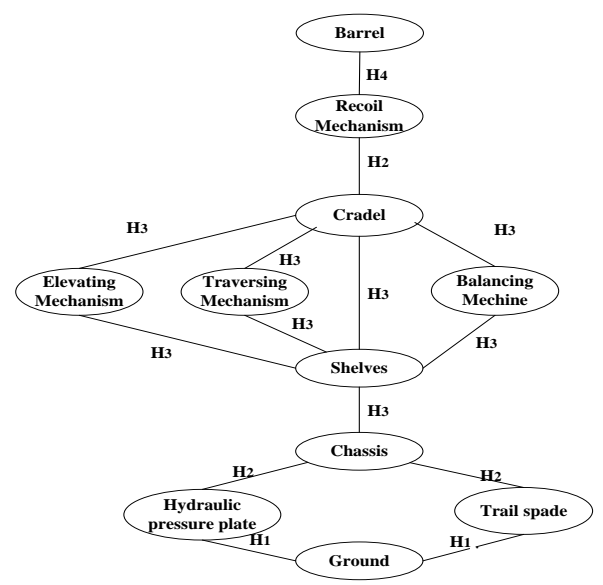

Fig.2 Vehicle artillery topology

H1-plane contact,H2-fixed vice,H3-rotation vice,H4-translation vice

\section{Recoil Analysis}

In the shooting process, the gun body in the gunpowder gas driven along the cradle for recoil and reentry movement. In this process, the artillery is mainly acted by the gun bore force $F_{p t}$ and the role of the back seat resistance $R$ in the direction of the gun body ${ }^{[3]}$.The formula for the coercive force is:

During the movement in the chamber,

$$
F_{p t}=\frac{1}{\alpha}\left(1+\frac{1}{2} \cdot \frac{\omega}{q}\right) \cdot S \cdot p
$$

Postponement of the gunpowder gas,

$$
F_{p t}=\chi^{F_{g} \bullet \frac{t-t_{g}}{b}}
$$

After postponement,

$$
F_{p t}=0
$$

where: $\alpha$-- the secondary work coefficient;

$\omega$-- the quality of the charge;

$q$-- the quality of the projectile;

$S$-- the gun bore cross-sectional area;

$p$--the average pressure of gunpowder gas;

$\chi$-- the muzzle brake device impulse characteristics;

$b$--the time constant of the gunpowder gas;

$t_{g}$--the moment when the projectile flew away from the muzzle.

The recoil resistance $R$ is mainly caused by the recoil mechanism and recuperator, including the recuperator force $F_{f j}$, the recoil force $\phi_{0}$ and the constant friction $R_{\varphi}$.

$$
R=F_{f j}+\phi_{0}+R_{\varphi}
$$

In the process of recoil, the relationship of between the return force and artillery recoil trip can be expressed as:

$$
F_{f j}=50000 \times\left(\frac{2.15}{2.15-x_{2}}\right)^{1.3}
$$


Where: $x_{2}-$ - the artillery of the recoil ride.

In the process of recoil, the recoil force can be expressed as a function of the speed of the gun recoil:

$$
\begin{aligned}
& \phi_{0}=\left(1800 \times \frac{150+a_{x}}{a_{x}^{2}}+130\right) \dot{x}_{2}^{2} \\
& a_{x}=\left(23-\frac{\pi}{4} d_{x}^{2}\right)
\end{aligned}
$$

Where: $a_{x}$-- the flow mouth area of recoil mechanism;

$\dot{x}_{2}$-- the gun recoil speed.

The constant friction $R_{\varphi}$ consists of the frictional force generated by the recoil device during the recoil, the frictional force between the rear seat and the cradle, and the gravitational component in the recoil direction, which can be expressed as:

$$
\begin{aligned}
& R_{\varphi}=f \cdot M g \cdot \cos \varphi-M g \cdot \sin \varphi+F_{0} \\
& F_{0}=F_{01}+F_{02}
\end{aligned}
$$

Where: $M$-- the quality of the recoil part of the artillery;

$g$-- the acceleration of gravity;

$f$-- the friction coefficient between the recoil part and the cradle;

$\varphi$--the gun angle;

$F_{0}$-- the frictional force caused by the anti-recoil device plug;

$F_{01}$-- the frictional force generated by the recuperator;

$F_{02}$--the frictional force generated by the recoil mechanism .

In summary, artillery recoil can be expressed as:

$$
F=F_{p t}-R=F_{p t}-F_{f j}-\phi_{0}-R_{\varphi}
$$

Considering the movement and the force in the shooting process are complex, this paper uses Fortran program to define the force acted on the artillery during the process of recoil, and transfers this procedure in the simulation process ${ }^{[4]}$.

\section{Simulation Results and Analysis}

In this paper, the vibration of the body barrel of a vehicle cannon is simulated under the condition of $0{ }^{\circ}$ in the vertical direction and $0{ }^{\circ}$ in the horizontal direction, and the low between the change of the maximum amplitude of the barrel and the soil stiffness or damping is obtained. The recoil motion curve of the gun is shown in Fig.3, and the maximum displacement is $728 \mathrm{~mm}$, and the reciprocal movement is completed in about $0.8 \mathrm{~s}$, which is consistent with the actual recoil movement of the gun.

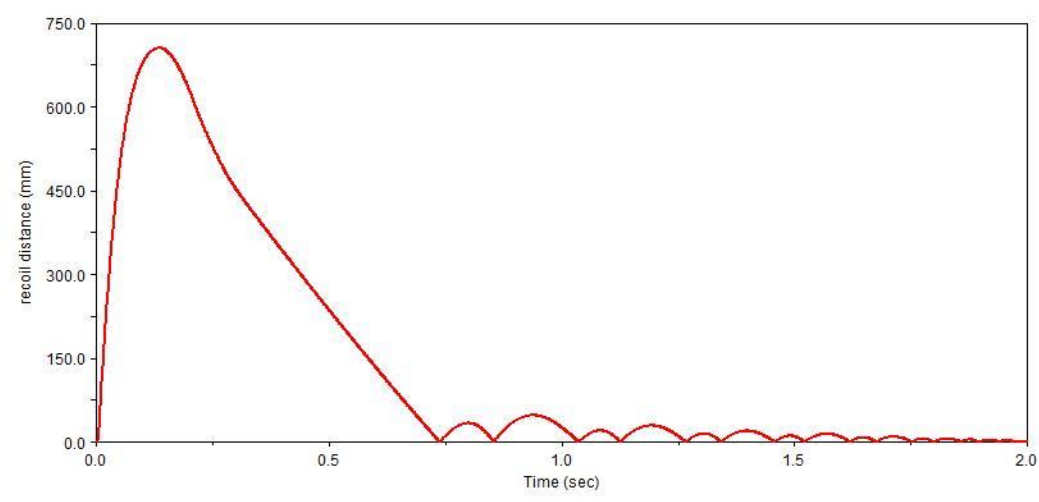

Fig.3 Artillery recoil curve 
Changing the stiffness coefficient $\mathrm{K}$ under the damping coefficient $\mathrm{C}=20,30,50$ respectively , the vibration of the gun under different soil conditions is obtained. The results are shown in Table 1 and table2.

Table 1. gun longitudinal vibration amplitude

\begin{tabular}{|c|c|c|c|c|c|c|c|c|}
\hline $\mathrm{k}$ & 0 & 500 & 1000 & 2000 & 4000 & 6000 & 8000 & 10000 \\
\hline $\mathrm{C}=20$ & 4.4459 & 4.4638 & 4.496 & 6.2922 & 8.3194 & 11.7653 & 12.6783 & 12.7874 \\
\hline $\mathrm{C}=30$ & 4.5046 & 4.5219 & 4.6122 & 5.0526 & 7.4875 & 10.4991 & 11.4194 & 11.6930 \\
\hline $\mathrm{C}=50$ & 4.6264 & 5.0693 & 5.7681 & 5.0693 & 7.0391 & 8.8120 & 9.7015 & 10.0687 \\
\hline
\end{tabular}

Table 2. Ruler lateral vibration amplitude

\begin{tabular}{|c|c|c|c|c|c|c|c|c|}
\hline $\mathrm{k}$ & 0 & 500 & 1000 & 2000 & 4000 & 6000 & 8000 & 10000 \\
\hline $\mathrm{C}=20$ & 1.4503 & 1.8496 & 2.1674 & 3.1781 & 6.4267 & 6.4267 & 5.3243 & 3.779 \\
\hline $\mathrm{C}=30$ & 1.5567 & 1.8949 & 2.1773 & 2.6224 & 4.5309 & 5.0978 & 4.1909 & 3.3095 \\
\hline $\mathrm{C}=50$ & 1.8791 & 2.0997 & 2.2862 & 2.0096 & 2.6582 & 3.3122 & 3.1125 & 2.5471 \\
\hline
\end{tabular}

This paper uses MATLAB software to fit the data in the above table, the results shown in Fig4 and Fig.5. It can be seen from Fig.4 that the maximum amplitude of the longitudinal vibration increases with the increase of the soil rigidity $\mathrm{k}$ and decreases with the increase of the damping and tends to be stable finally. As shown in Fig.5,the amplitude of the transverse vibration increases first and then decreases with the increase of soil stiffness $\mathrm{k}$, and decreases with the increase of damping c.

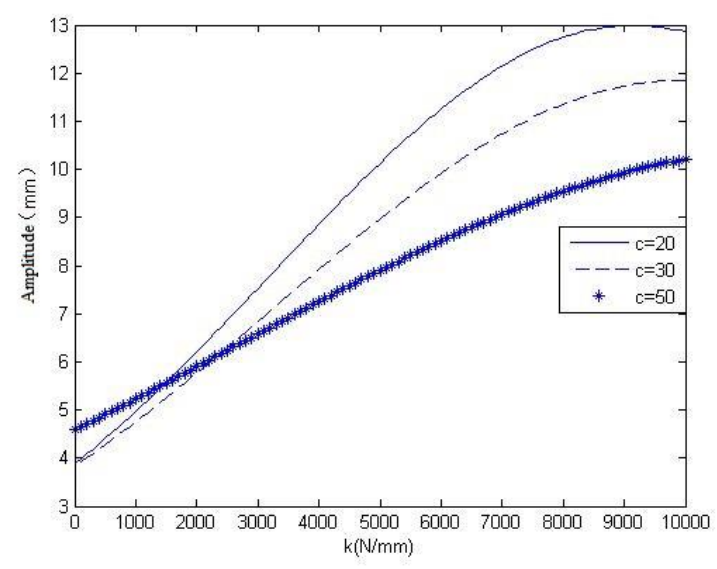

Fig.4 Longitudinal vibration curve of the barrel

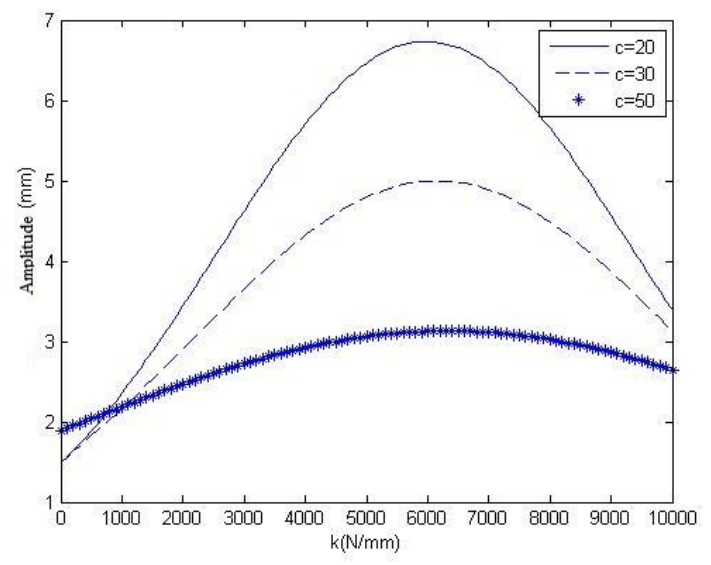

Fig.5 Transverse vibration curve of the barrel

\section{Summary}

In this paper, the virtual prototype model of the vehicle artillery is established and the deformation of the soil is simulated by the spring damper. The simulation data are processed by matlab under the condition of changing the stiffness coefficient and the damping coefficient. And the law of the influence of the soil property on the muzzle vibration has a certain reference value to the follow-up study.

\section{Acknowledgements}

This work was financially supported by the Hebei Province Science and Technology Project (15210701D). 


\section{References}

[1] Cai Wenyong, Chen Yunsheng, Yang Guolai. Dynamic simulation of vehicle artillery [J]. Journal of Fireworks Launch and Control, 2006: 12-15.

[2] Zuo Yanrui, Zong Zhijian, Cheng Yuan, etc. Modeling and Application of Soil Dynamics in Vehicle Ground Mechanics [J]. Journal of Lanzhou University, 2009,45 (5): 110-114.

[3] Di Changchun, Yang Yuliang, Qin Junqi, etc.Study on impact parameters of gun recoil test equipment [J].Journal of Artillery Launch \& Control, 2012 (02): 25-28.

[4] Jia Changzhi, Wang Xinggui, Qin Junqi, etc. Modeling and Simulation Analysis of Gun System Based on Virtual Prototype [J]. Journal of Vibration and Shock, 2001,20 (4): 4-9. 